\title{
ANÁLISE RADIOGRÁFICA COMPARATIVA DA CIFOSE JUNCIONAL ENTRE INSTRUMENTAÇÃO HÍBRIDA, GANCHOS E PARAFUSOS NA ESCOLIOSE IDIOPÁTICA DO ADOLESCENTE
}

\author{
COMPARATIVE RADIOGRAPHIC ANALYSIS OF JUNCTIONAL KYPHOSIS BETWEEN HYBRID \\ INSTRUMENTATION, HOOKS AND SCREWS IN ADOLESCENT IDIOPATHIC SCOLIOSIS
}

\author{
ANÁLISIS RADIOGRÁFICO COMPARATIVO DE LA CIFOSIS DE UNIÓN ENTRE LOS \\ INSTRUMENTOS HÍBRIDOS, LOS GANCHOS Y LOS TORNILLOS \\ EN ESCOLIOSIS IDIOPÁTICA DEL ADOLESCENTE
}

Hans Grohs ${ }^{1}$, Luciano Antonio Nassar Pellegrino², Ricardo Shigueaki Galhego Umeta², Maria Fernanda Silber Caffaro³,

Robert Meves ${ }^{4}$, Élcio Landim ${ }^{5}$, Osmar Avanzi ${ }^{6}$

\begin{abstract}
RESUMO
Objetivo: Avaliação radiográfica das cifoses juncionais proximal e distal em pacientes submetidos a artrodese e diferentes tipos de instrumentação posterior no tratamento cirúrgico da escoliose idiopática do adolescente (EIA). Método: Foi realizado estudo retrospectivo com avaliação radiográfica de 34 pacientes submetidos à artrodese da coluna vertebral com instrumentação posterior, sendo 10 com ganchos (Grupo I), 13 com ganchos e parafusos (Grupo II) e 11 com parafusos (Grupo III), entre junho de 1997 e dezembro de 2009 . Foi avaliada a ocorrência de cifose juncional proximal (CJP) e cifose juncional distal (CJD) à artrodese, no pré-operatório, no pós-operatório imediato e no final do seguimento, pós-operatório tardio, que foi de, no mínimo, 12 meses. Resultados: Os pacientes do grupo I apresentaram cifose torácica de menor valor no pré-operatório, porém a lordose lombar permaneceu inalterada ao longo da evolução. Os pacientes do grupo II e grupo III apresentaram aumento do valor aferido da lordose lombar no pós-operatório. Não houve diferença significativa para a ocorrência de cifose juncional proximal entre os três grupos estudados. Com relação à cifose juncional distal, houve aumento estatisticamente significativo do valor entre pré é pós-operatório, para os grupos II e III. Conclusão: A avaliação radiográfica das cifoses juncionais proximal e distal em pacientes submetidos à artrodese e diferentes tipos de instrumentação no tratamento cirúrgico da EIA revelou a presença de cifoses juncionais proximais pré-operatórias, que não evoluíram para a deformidade juncional pós-operatória, além de ausência completa da anormalidade juncional distal
\end{abstract}

Descritores: Coluna vertebral/patologia; Escoliose/cirurgia, Fusão espinhal/instrumentação; Adolescente.

\begin{abstract}
Objective: Radiographic evaluation of proximal and distal junctional kyphosis in patients undergoing spinal fusion and different types of posterior instrumentation, in the surgical treatment for adolescent idiopathic scoliosis (AIS). Method: A retrospective review was done with radiographic evaluation of 34 patients who were submitted to spinal fusion with posterior instrumentation, divided as follows: 10 using only hooks (Group I), 13 hybrid fixation (screws and hooks) (Group II) and 11 using only pedicle screws (Group III) from June 1997 to December 2009. The study assessed the occurrence of junctional kyphosis both proximal and distal to the arthrodesis, in the preoperative, immediate postoperative periods, in the final follow up, and also in the late postoperative period, which was at least 12 months. Results: Patients in group I showed lower value of thoracic kyphosis preoperatively, but the lumbar lordosis remained unchanged throughout evolution. Patients in group II and group III showed an increase in the assessed value of lumbar lordosis postoperatively. There was no significant difference in the occurrence of proximal junctional kyphosis among the three groups. Regarding the distal junctional kyphosis, there was a statistically significant increase in value between the preoperative and postoperative for groups II and III. Conclusion: Radiographic evaluation of the proximal and distal junctional kyphosis in patients who underwent arthrodesis and different types of instrumentation in the surgical treatment of adolescent idiopathic scoliosis, revealed the presence of preoperative proximal junctional kyphosis, which have not progressed to postoperative junctional deformity, and also complete absence of distal junctional abnormality.
\end{abstract}

Keywords: Spine/pathology; Scoliosis/surgery, Spinal fusion/instrumentation; Adolescent.

\section{RESUMEN}

Objetivo: Evaluación radiográfica de cifosis por unión proximal y distal en pacientes sometidos a artrodesis y diferentes tipos de instrumentación como tratamiento quirúrgico de escoliosis idiopática del adolescente (EIA). Método: Se realizó un estudio retrospectivo de evaluación radiográfica de 34 pacientes sometidos a artrodesis de la columna con instrumentación posterior, 10 con ganchos (Grupo l), 13 con ganchos y tornillos (Grupo II) y 11 con tornillos (Grupo III), entre junio de 1997 y diciembre de 2009. Se evaluó la aparición de cifosis proximal y distal a la artrodesis en los períodos preoperatorio, postoperatorio inmediato y al final del seguimiento, período postoperatorio tardío, de

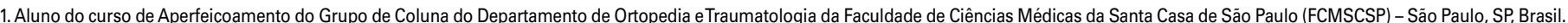
2. Pós-graduando do Grupo de Coluna do Departamento de Ortopedia e Traumatologia da Faculdade de Ciências Médicas da Santa Casa de São Paulo (FCMSCSP) - São Paulo, SP, Brasil.

3. Assistente e Professora Instrutora do Grupo de Coluna do Departamento de Ortopedia eTraumatologia da Irmandade Santa Casa de Misericórdia de São Paulo (ISCMSP) - São Paulo, SP, Brasil.

4. Chefe do Grupo de Coluna e Professor-assistente do Departamento de Ortopedia eTraumatologia da Irmandade Santa Casa de Misericórdia de São Paulo (ISCMSP) - São Paulo, SP, Brasil.

5. Consultor do Grupo de Coluna do Departamento de Ortopedia eTraumatologia da Faculdade de Ciências Médicas da Santa Casa de São Paulo (FCMSCSP) - São Paulo, SP, Brasil.

6. Professor Adjunto e Diretor do Departamento de Ortopedia eTraumatologia da Faculdade de Ciências Médicas da Santa Casa de São Paulo (FCMSCSP) - São Paulo, SP, Brasil.

Trabalho realizado no Grupo de Coluna do Departamento de Ortopedia eTraumatologia do Hospital da Santa Casa de Misericórdia de São Paulo - São Paulo, SP. Correspondência: Robert Meves. Rua Dr Cesário Mota Jr, 61, Sta Cecília São Paulo, SP, Brasil.01221-020. E-mail: coluna@ santacasasp.org.br. robertmeves@ hotmail.com 
por lo menos 12 meses. Resultados: Los pacientes del Grupo I presentaron menor valor de cifosis torácica preoperatoria, pero la lordosis lumbar se mantuvo sin cambios durante la evolución. Los pacientes en el grupo II y grupo III mostraron incremento del valor verificado de lordosis lumbar en el postoperatorio. No hubo diferencia significativa en la aparición de cifosis proximal a la unión entre los tres grupos. En cuanto a la cifosis por unión distal, se produjo un aumento estadísticamente significativo del valor entre preoperatorio y postoperatorio en los grupos II y III. Conclusión: La evaluación radiográfica de la cifosis por unión proximal y distal en pacientes sometidos a artrodesis y diferentes tipos de instrumentación como tratamiento quirúrgico de escoliosis idiopática del adolescente reveló la presencia preoperatoria de cifosis proximales a la unión , que no evolucionaron para deformidad postoperatoria por unión y además, ausencia completa de anormalidad de la unión distal.

Descriptores: Columna vertebral/patología; Escoliosis/cirugía; Fusión vertebral/ instrumentación; adolescentes.

\section{INTRODUÇÃO}

Sempre houve por parte dos cirurgiões, uma grande preocupação com as correções das deformidades no plano coronal das escolioses idiopáticas. Nos últimos anos tem se dado maior atenção para a correção global nos três eixos, o coronal, axial e sagital ${ }^{1}$.

O instrumental de terceira geração permitiu correção mais efetiva, com a qual se pode aplicar forças de compressão e distração assimetricamente resultando em um torque de força corretiva no plano coronal e sagital. Permite ainda mobilizar a vértebra que se encontra no ápice da curva levando a uma correção nos três planos².

As construções segmentares da coluna toracolombar baseadas em ganchos são mais rígidas do que suas antecessoras. Esse aumento da rigidez proporcionou aumento das taxas de artrodese sem necessitar do uso de órteses ou imobilização com gesso. Desvantagens dessa construção, tal qual com implantes rígidos, incluem degeneração discal e desequilíbrio sagital nos segmentos adjacentes. Devido a sua inserção no corpo vertebral, os parafusos permitem ao cirurgião aplicar forças de distração, compressão, rotação ou translação. Temos como vantagens do seu uso: aumento da taxa de artrodese, ganho e manutenção da redução, reabilitação precoce e torna desnecessário o uso de órteses.

Ao invés das construções com parafusos pediculares, que partilham de fixação nas três colunas, ganchos ancoram somente nos elementos posteriores e, portanto não têm o mesmo poder de correção da escoliose. Além disso, enquanto parafusos pediculares mal posicionados proporcionam riscos de lesão neurológica e vascular catastróficas, ganchos pela sua própria natureza são invasores do canal vertebral ${ }^{3}$.

A escoliose idiopática define uma doença musculoesquelética comum e potencialmente grave de etiologia desconhecida. Quando ocorre dos 10 aos 20 anos é definida como do adolescente.

Sua prevalência (uma curva maior do que 10 graus) na população infantil e adolescente foi relatada em torno de 0,5 a $3 \%^{4}$.

Um fenômeno relativamente comum em instrumentações mais longas é o desenvolvimento de cifoses juncionais proximais e distais ${ }^{1,5}$

A cifose juncional proximal é uma deformidade cifótica nos segmentos móveis imediatamente cefálicos aos segmentos artrodesados ${ }^{5}$.

De acordo com Glattes et al. ${ }^{6}$, a cifose juncional proximal é definida como valor angular pré-operatório $>10^{\circ}$ e aumento de no mínimo $10^{\circ}$ no valor angular final em relação ao pré-operatório.

Fatores de risco para cifose juncional proximal: diminuição da cifose torácica pós-operatória (diminuição $>5^{\circ}$ ), toracoplastia associada; sexo masculino; instrumentação híbrida (ganchos proximais e parafusos distais); valor angular torácico (T5-T12>40 $\left.{ }^{\circ}\right)^{7}$.

A cifose juncional distal é uma deformidade cifótica nos segmentos móveis imediatamente caudais aos segmentos artrodesados ${ }^{5}$.

De acordo com Lowe et al. ${ }^{8}$, a cifose juncional distal é definida como valor angular igual ou superior a $10^{\circ}$ no pós-operatório.

Fatores de risco para cifose juncional distal: instrumentação posterior; cifose toracolombar pré-operatória; o término da instrumentação ao nível da vértebra terminal inferior de Cobb ao invés de 1 ou 2 níveis abaixo, incluindo o segmento cifótico pré-operatório; a não manutenção ou ganho de um perfil sagital normal sobre a região torácica principal (T5-T12) e junção toracolombar (T12-L1) 8-11.

A incapacidade de restaurar ou manter o equilíbrio sagital global após artrodeses lombares pode resultar em dor e resultado funcional insatisfatório ${ }^{7}$.
A perda da lordose lombar normal leva a uma diminuição do braço de alavanca dos músculos paraespinhais e consequentemente maiores forças são necessárias para manter a postura ereta. A cifose juncional determina grande sobrecarga nos músculos, ligamentos e discos da coluna. Distúrbios mínimos no alinhamento sagital podem ser compensados por ação muscular para manter o nível de visão ${ }^{12}$.

A descompensação progressiva é um processo gradual de falha nos mecanismos musculares (estabilizadores dinâmicos) para manter a postura, seguido de falha gradual das estruturas capsulares e ligamentares (estabilizadores rígidos). Isto cria uma situação de deformidade progressiva com dor e limitação da função para o paciente ${ }^{13}$.

O objetivo do estudo foi analisar radiograficamente a incidência de cifose juncional proximal e distal após o tratamento cirúrgico da EIA em relação aos diferentes tipos de instrumentação.

\section{MATERIAL E MÉTODO}

Após aprovação do estudo pelo comitê de ética e pesquisa, protocolo de pesquisa CEP 106/10, foram analisados, retrospectivamente, os prontuários e as radiografias de pacientes portadores de escoliose idiopática do adolescente (EIA) submetidos à correção cirúrgica com instrumental de terceira geração com ganchos (Grupo I), com ganchos e parafusos (Grupo II) e somente com parafusos (Grupo III) no período de junho de 1997 a dezembro de 2009.

Foram analisadas as radiografias em perfil ortostáticas do momento pré-operatório (pré-op.), pós-operatório imediato (pós-op. imediato) e no pós-operatório tardio (pós-op. tardio), que foi de, no mínimo, 12 meses.

Critérios de inclusão: pacientes portadores de EIA, operados somente pela via posterior no período de junho de 1997 a dezembro 2009 com instrumental de terceira geração.

Critérios de exclusão: pacientes abordados por via anterior e posterior; incapacidade de visualizar adequadamente os corpos vertebrais devido a radiografias de má qualidade, radiografias insuficientes e período de segmento inferior a 12 meses.

As indicações cirúrgicas foram baseadas no valor angular mensurado nas radiografias (ortostática anteroposterior e perfil, inclinações laterais e tração) pelo método de Cobb, maturidade esquelética dos pacientes, distúrbios ventilatórios, compensação do tronco, deformidade estética e critérios de progressão inaceitáveis documentados em radiografias. Para a seleção do nível de artrodese a ser realizado em cada caso, utilizamos a classificação de King et al. ${ }^{14}$

Foi realizada instrumentação e artrodese por via posterior, com adição de enxerto autólogo retirado da crista ilíaca. A instrumentação do grupo I foi feita através da montagem de múltiplos ganchos sobre duas hastes longitudinais e duas hastes transversais, não sendo realizada manobra derrotativa. Não se utilizou qualquer imobilização pós-operatória. O grupo II foi composto por ganchos e parafusos pediculares associados a duas hastes longitudinais moldadas e duas hastes transversais. No grupo III, o instrumental foi exclusivamente composto de parafusos pediculares associados a duas hastes longitudinais e duas transversais. Nos três grupos foi realizada a manobra de translação para a correção da deformidade.

O grupo I foi composto por 10 pacientes, o grupo II por 13 e por 11 pacientes no grupo III. 


\section{Dados demográficos e classificação das curvas.}

Do total de 34 pacientes, seis eram do sexo masculino (17\%) e 28 femininos (83\%). A média de idade na ocasião da operação foi de 15,35 anos (variando de 12 a 23 anos). A média de vértebras artrodesadas foi 10,47 (variando de 8 a 14). De acordo com a classificação de King, 11 foram classificados King II, 20 King III, 1 King IV, 1 King V. Em um paciente não foi possível a classificação por King por se tratar de curva torácica à esquerda. A classificação foi realizada pelo Grupo de Cirurgia de Coluna da Santa Casa de Misericórdia de São Paulo.

\section{Mensurações radiográficas}

A mensuração incluiu: medida da junção proximal (MJP; ângulo de Cobb entre a vértebra mais proximal instrumentada e a segunda vértebra acima desta) ${ }^{1,5-7,15}$, cifose torácica (T5-T12) ${ }^{16}$, lordose lombar (L1-L5) ${ }^{16}$ e medida da junção distal (MJD; ângulo de Cobb entre a vértebra mais distal instrumentada e a primeira vértebra abaixo desta $)^{9-11}$. Foram utilizados valores positivos para cifose e negativos para lordose.

\section{ANÁLISE ESTATÍSTICA}

Para responder o objetivo do estudo as medidas foram descritas ao longo do tempo segundo instrumentação com uso de medidas resumo e comparadas entre os momentos e instrumentações com uso de análises de variâncias (ANOVA) com medidas repetidas com dois fatores, sendo a instrumentação (grupo I, grupo II e grupo III) o fator fixo e o momento (pré-operatório, pós-operatório imediato e pós-operatório tardio) o fator de repetição. Foi suposta matriz de correlações autorregressiva de ordem um entre os momentos de avaliação. Após a realização das análises foram realizadas comparações múltiplas de Tukey para verificar em quais instrumentações ou momentos há diferença quando a ANOVA apresentou significância.

Os resultados foram ilustrados com uso de gráficos de perfis médios com os respectivos erros padrões e os testes foram realizados com nível de significância de 5\%.

\section{RESULTADOS}

Para elaboração deste estudo foram observados 34 adolescentes com EIA, sendo que 10 pacientes fizeram instrumentação com ganchos, 13 com ganchos e parafusos e $11 \mathrm{com}$ parafusos pediculares.

Os valores de cifose torácica para o grupo II e grupo III não apresentaram diferenças relevantes entre o pré e pós-operatório tardio. Os pacientes do grupo I possuíam menores valores de cifose pré-op. e na evolução pós-cirúrgica demonstraram um aumento da medida torácica. Os dados não apresentaram relevância estatística (Figura 1).

A lordose lombar dos pacientes do grupo I permaneceu quase inalterada, ao contrário dos grupos II e III que, no pós-op. imediato, aumentaram o valor sensivelmente e o mantiveram com uma pequena perda no pós-operatório tardio. Os dados foram estatisticamente relevantes (Figura 2).

A medida juncional proximal do grupo I não apresentou diferença estatística entre os momentos pré-operatório e pós-operatório, apesar da pequena diminuição no pós-operatório imediato. O grupo II permaneceu quase inalterado. O grupo III apresentou um decréscimo da medida já no pós-op. imediato que se manteve ao longo do tempo (Figura 3).

Ocorreram dois casos de cifose juncional proximal (CJP) pré-operatória no grupo I (20\%). Já no Grupo II ocorreram três casos de CJP (23\%) sendo todos os casos de cifose pré-operatória. Os 11 pacientes submetidos a instrumentação com uso exclusivo de parafusos (Grupo III) apresentaram três casos de CJP pré-operatória (27\%). Não houve significância estatística

Para os valores de medida juncional distal, os três grupos apresentaram já no pós-op imediato um aumento, que foi maior proporcionalmente nos grupos II e III e se manteve crescente ao longo do período analisado para todos os grupos (Figura 4).

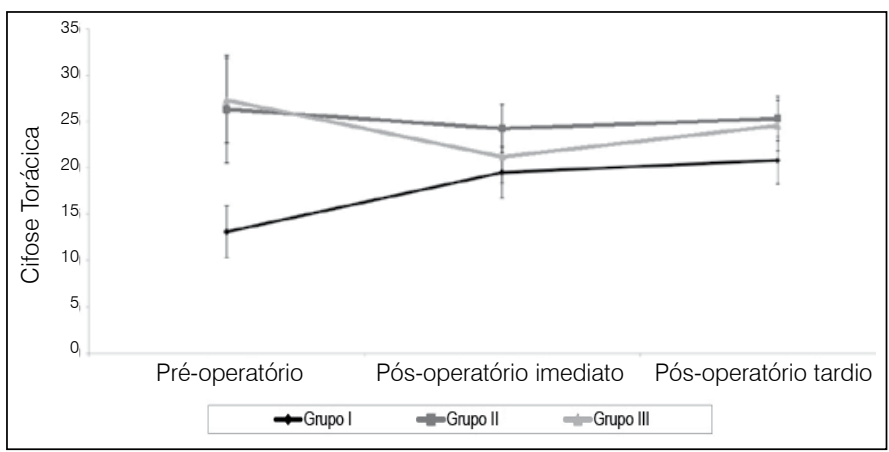

Figura 1. Evolução da medida dos valores de cifose torácica ao longo do tempo (pré-operatório; pós-operatório imediato; pós-operatório tardio) para cada grupo e seus respectivos erros padrões. $\mathrm{P}=0,112$.

Fonte: SAME Hospital da Irmandade Santa Casa de Misericórdia de São Paulo.

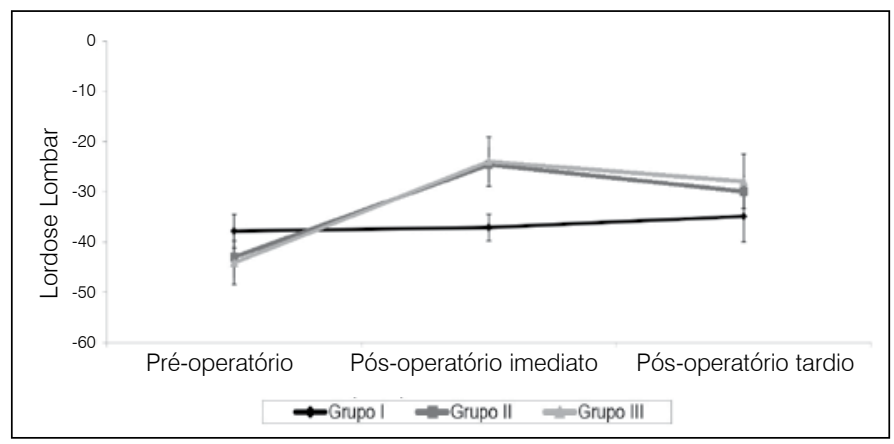

Figura 2. Evolução da medida dos valores de lordose lombar ao longo do tempo (pré-operatório; pós-operatório imediato; pós-operatório tardio) para cada grupo e seus respectivos erros padrões. $P=0,003$.

Fonte: SAME Hospital da Irmandade Santa Casa de Misericórdia de São Paulo.

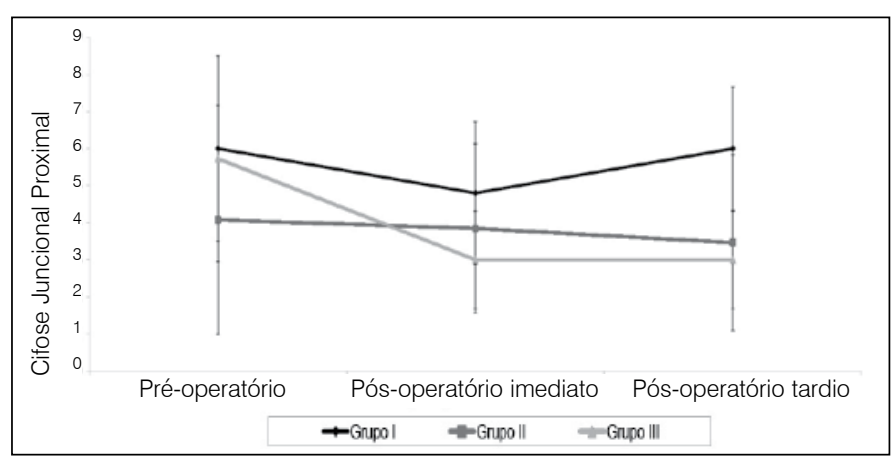

Figura 3. Evolução da medida dos valores de cifose juncional proximal ao longo do tempo (pré-operatório; pós-operatório imediato; pós-operatório tardio) para cada grupo e seus respectivos erros padrões. $\mathrm{P}=0,794$. Fonte: SAME Hospital da Irmandade Santa Casa de Misericórdia de São Paulo.

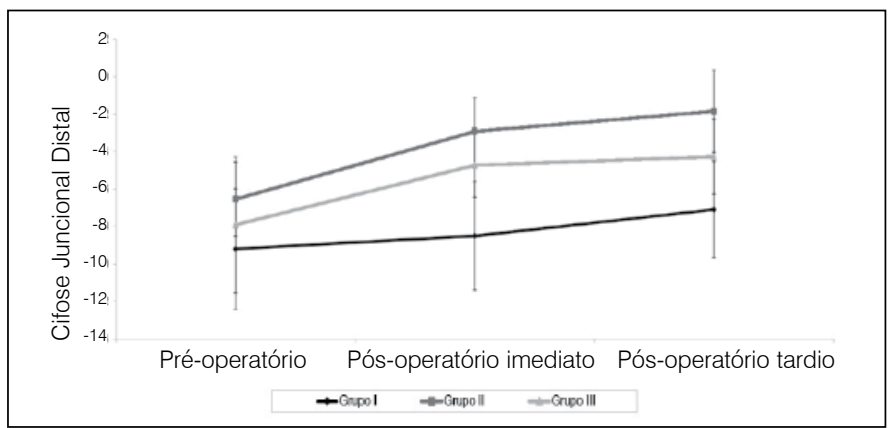

Figura 4. Evolução da medida dos valores de cifose juncional distal ao longo do tempo (pré-operatório; pós-operatório imediato; pós-operatório tardio) para cada grupo e seus respectivos erros padrões. $P=0,841$.

Fonte: SAME Hospital da Irmandade Santa Casa de Misericórdia de São Paulo. 
Não houve casos de CJD nos três grupos e não houve relevância estatística.

Foi realizado o cálculo da média e desvio padrão das medidas de cifose torácica, lordose lombar, cifoses juncionais proximal e distal para cada grupo nos diferentes momentos (Tabela 1).

A cifose torácica e a cifose juncional proximal não apresentam alteração média estatisticamente significativa entre os momentos de avaliação e nem entre os tipos de instrumentação ( $p>0,05)$. A lordose lombar apresenta comportamento médio entre as instrumentações estatisticamente diferentes ao longo dos momentos ( $p=0,003)$ e a cifose juncional distal apresenta em média alteração entre os momentos avaliados independente da instrumentação $(p=0,039)$ (Tabela 2).

Tem-se que nos grupos II e grupo III há aumento médio da lordose lombar estatisticamente significativo do pré-operatório para o pós-operatório imediato ( $p<0,001$ e $p<0,001$ respectivamente) e apenas no grupo III há aumento médio estatisticamente significativo da lordose lombar entre o pré-operatório e o pós-operatório tardio $(p=0,020)$ (Tabela 3).

A cifose juncional distal aumenta em média do pré-operatório para o pós-operatório, tanto recente como tardio $(p=0,050$ e $p=0,038)$ para todas as instrumentações (Tabela 4).

Tabela 1. Descrição das medidas realizadas segundo momentos e instrumentação.

\begin{tabular}{|c|c|c|c|c|c|c|c|c|c|c|}
\hline \multirow{3}{*}{ Variável } & \multirow{3}{*}{ Momento } & \multicolumn{9}{|c|}{ Instrumentação } \\
\hline & & \multicolumn{3}{|c|}{ Grupo I } & \multicolumn{3}{|c|}{ Grupo II } & \multicolumn{3}{|c|}{ Grupo III } \\
\hline & & Média & DP & $n$ & Média & DP & $\mathbf{n}$ & Média & DP & $\mathrm{N}$ \\
\hline \multirow{3}{*}{ Cifose Torácica } & pré-operatório & 13,10 & 8,79 & 10 & 26,31 & 20,85 & 13 & 27,27 & 15,15 & 11 \\
\hline & pós-op. imediato & 19,50 & 8,77 & 10 & 24,23 & 9,48 & 13 & 21,18 & 9,43 & 11 \\
\hline & pós-op. tardio & 20,80 & 8,08 & 10 & 25,31 & 8,63 & 13 & 24,55 & 8,98 & 11 \\
\hline \multirow{3}{*}{ Lordose Lombar } & pré-operatório & $-37,80$ & 10,55 & 10 & $-43,00$ & 14,91 & 13 & $-44,09$ & 14,31 & 11 \\
\hline & pós-op. imediato & $-37,10$ & 8,43 & 10 & $-24,62$ & 12,84 & 13 & $-24,00$ & 16,28 & 11 \\
\hline & pós-op. tardio & $-34,90$ & 16,04 & 10 & $-29,92$ & 11,54 & 13 & $-27,91$ & 17,93 & 11 \\
\hline \multirow{3}{*}{$\begin{array}{l}\text { Medida } \\
\text { Juncional } \\
\text { Proximal }\end{array}$} & pré-operatório & 6,00 & 7,89 & 10 & 4,08 & 11,12 & 13 & 5,73 & 9,19 & 11 \\
\hline & pós-op. imediato & 4,80 & 6,07 & 10 & 3,85 & 8,23 & 13 & 3,00 & 4,36 & 11 \\
\hline & pós-op. tardio & 6,00 & 5,27 & 10 & 3,46 & 8,55 & 13 & 3,00 & 4,36 & 11 \\
\hline \multirow{3}{*}{$\begin{array}{c}\text { Medida } \\
\text { Juncional Distal }\end{array}$} & pré-operatório & $-9,20$ & 10,20 & 10 & $-6,54$ & 7,14 & 13 & $-7,91$ & 12,07 & 11 \\
\hline & pós-op. imediato & $-8,50$ & 9,17 & 10 & $-2,92$ & 6,47 & 13 & $-4,73$ & 5,69 & 11 \\
\hline & pós-op. tardio & $-7,10$ & 8,13 & 10 & $-1,85$ & 7,94 & 13 & $-4,27$ & 6,63 & 11 \\
\hline
\end{tabular}

Fonte: SAME Hospital da Irmandade Santa Casa de Misericórdia de São Paulo.

Tabela 2. Resultado das análises de variância para comparação de cada medida entre instrumentações e momentos.

\begin{tabular}{c|c|c}
\hline Variável & Fator & $\mathbf{p}$ \\
\hline \multirow{3}{*}{ Cifose Torácica } & Instrumentação & 0,159 \\
\cline { 2 - 3 } & Momento & 0,575 \\
\cline { 2 - 3 } & Instrumentação* Momento & 0,112 \\
\hline \multirow{3}{*}{ Lordose Lombar } & Instrumentação & 0,588 \\
\cline { 2 - 3 } & Momento & $<0,001$ \\
\cline { 2 - 3 } & Instrumentação*Momento & 0,003 \\
\hline \multirow{3}{*}{ CJP } & Instrumentação & 0,786 \\
\cline { 2 - 3 } & Momento & 0,358 \\
\cline { 2 - 3 } & Instrumentação*Momento & 0,794 \\
\hline \multirow{3}{*}{ CJD } & Instrumentação & 0,326 \\
\cline { 2 - 3 } & Momento & 0,039 \\
\cline { 2 - 3 } & Instrumentação*Momento & 0,841 \\
\hline
\end{tabular}

Fonte: SAME Hospital da Irmandade Santa Casa de Misericórdia de São Paulo.
Tabela 3. Resultado das comparações múltiplas para Lordose Lombar entre momentos e instrumentação.

\begin{tabular}{|c|c|c|c|c|c|}
\hline $\begin{array}{c}\text { Momento / } \\
\text { Instrumentação }\end{array}$ & \multicolumn{2}{|c|}{ Comparação } & \multirow{2}{*}{\begin{tabular}{|c|}
$\begin{array}{c}\text { Diferença média } \\
\text { estimada }\end{array}$ \\
5,20
\end{tabular}} & \multirow{2}{*}{\begin{tabular}{|c|} 
Erro Padrão \\
5,65
\end{tabular}} & \multirow{2}{*}{$\frac{\mathbf{p}}{0,991}$} \\
\hline \multirow{3}{*}{ pré-operatório } & Grupo 1 & Grupo II & & & \\
\hline & Grupo I & Grupo III & 6,29 & 5,87 & 0,976 \\
\hline & Grupo II & Grupo III & 1,09 & 5,51 & 1,000 \\
\hline \multirow{3}{*}{ pós-operatório imediato } & Grupo I & Grupo II & $-12,48$ & 5,65 & 0,414 \\
\hline & Grupo I & Grupo III & $-13,10$ & 5,87 & 0,400 \\
\hline & Grupo II & Grupo III & $-0,62$ & 5,51 & 1,000 \\
\hline \multirow{3}{*}{ pós-operatório tardio } & Grupo I & Grupo II & $-4,98$ & 5,65 & 0,993 \\
\hline & Grupo I & Grupo III & $-6,99$ & 5,87 & 0,956 \\
\hline & Grupo II & Grupo III & $-2,01$ & 5,51 & 1,000 \\
\hline \multirow{3}{*}{ Grupo I } & pré-operatório & pós-op. imediato & $-0,70$ & 3,78 & 1,000 \\
\hline & pré-operatório & pós-op. tardio & $-2,90$ & 4,79 & 1,000 \\
\hline & pós-op. imediato & pós-op. tardio & $-2,20$ & 3,78 & 1,000 \\
\hline \multirow{3}{*}{ Grupo II } & pré-operatório & pós-op. imediato & $-18,38$ & 3,32 & $<0,001$ \\
\hline & pré-operatório & pós-op. tardio & $-13,08$ & 4,20 & 0,065 \\
\hline & pós-op. imediato & pós-op. tardio & 5,31 & 3,32 & 0,802 \\
\hline \multirow{3}{*}{ Grupo III } & pré-operatório & pós-op. imediato & $-20,09$ & 3,61 & $<0,001$ \\
\hline & pré-operatório & pós-op. tardio & $-16,18$ & 4,57 & 0,020 \\
\hline & pós-op. imediato & pós-op. tardio & 3,91 & 3,61 & 0,975 \\
\hline
\end{tabular}

Fonte: SAME Hospital da Irmandade Santa Casa de Misericórdia de São Paulo.

Tabela 4. Resultado das comparações múltiplas para cifose juncional distal entre momentos

\begin{tabular}{c|c|c|c|c}
\hline \multicolumn{2}{c|}{ Comparação } & Diferença média estimada & Erro Padrão & p \\
\hline pré-operatório & pós-op. recente & $-2,62$ & 1,09 & 0,050 \\
\hline pré-operatório & pós-op. tardio & $-3,59$ & 1,43 & 0,038 \\
\hline pós-op. recente & pós-op. tardio & $-0,97$ & 1,09 & 0,650 \\
\hline
\end{tabular}

Fonte: SAME Hospital da Irmandade Santa Casa de Misericórdia de São Paulo.

\section{DISCUSSÃO}

A cifose juncional proximal (CJP) tem sido observada com pouca atenção na evolução da EIA tratada com artrodese mais instrumentação posterior. Pouco se sabe sobre o seu desenvolvimento, mas apesar de não ser uma condição dolorosa, nem causar limitação, deve ser encarada como uma deformidade. Segundo Helgenson et al. ${ }^{1}$, o nível adjacente proximal foi mais cifótico nas construções que apenas utilizaram parafusos pediculares e houve uma tendência a diminuição da cifose com a colocação de ganchos na vértebra superior instrumentada. Ao contrário de Glattes et al. ${ }^{6}$ que afirmaram não haver característica específica do paciente ou técnica de instrumentação que fosse associada a CJP . Em nosso estudo, onde o grupo I (ganchos) apresentou uma tendência a maiores valores cifóticos, mas não foi estatisticamente significante, portanto os resultados foram semelhantes entre os três grupos.

Glattes et al. ${ }^{6}$ demonstraram que a CJP era mais comum quando a instrumentação terminava em T3. Sete casos, quatro no grupo II e três no Grupo III possuíam artrodese até T3, porém em nenhum desses apresentou-se o fenômeno estudado.

A prevalência de CJP, segundo Kim et al. ${ }^{7}$, em EIA após artrodeses por três instrumentações segmentares diferentes via posterior foi de $27 \%$ em um seguimento de dois anos. O seguimento dos pacientes em nosso estudo aconteceu durante um ano, restrições à parte, pela falta de radiografias ou na maioria das vezes pela má qualidade dos filmes, que dificultava a medição juncional na coluna torácica proximal. A ocorrência de CJP se restringiu ao período pré-operatório em oito casos nos três grupos (23\%). Não houve casos nos três grupos que cumprissem os dois critérios propostos (cifose juncional pré-op. igual ou superior a $10^{\circ}$ e progressão acima de $10^{\circ}$ 
no pós-op. $)^{6,7}$ e que caracterizassem a anormalidade pesquisada. Para a análise da cifose torácica, os valores foram menores, no pré-operatório, para os pacientes submetidos a instrumentação por ganchos (Grupo I). Não houve significância estatística entre os diferentes tipos e os momentos diferentes de comparação da cifose.

Segundo Kim et al. ${ }^{7}$, a hipercifose torácica $\left(>40^{\circ}\right)$ constituiria em um fator de risco para a CJP.

Lowe et al. ${ }^{8}$, em seu estudo de cifose juncional distal, destacaram a necessidade de inclusão do segmento juncional, quando a CJD está presente no pré-operatório a fim de evitar a deformidade juncional distal pela via posterior. A incidência de CJD pela instrumentação via posterior (14,7\%) foi o dobro da instrumentação pela via anterior $(7,5 \%)$.

Na nossa casuística não houve casos de CJD segundo a definição do autor, mesmo com o aumento do valor entre o pré-op e o pós-operatório, tanto recente como tardio $(p=0,050$ e $p=0,038)$ sendo estatisticamente significativo para todas as instrumentações.
Rhee et al. $^{16}$ afirmaram que a lordose lombar aumentou seu valor angular quando curvas lombares estruturadas foram instrumentadas via anterior ou posterior.

Na nossa casuística, a lordose lombar apresentou um aumento no valor médio no pós operatório recente para o grupo II e Grupo III ( $p<0,001$ e $p<0,001$ respectivamente) e tardio para o grupo III $(p=0,020)$.

\section{CONCLUSÃO}

A avaliação radiográfica das cifoses juncionais proximal e distal em pacientes submetidos à artrodese e diferentes tipos de instrumentação, no tratamento cirúrgico da escoliose idiopática do adolescente, revelou a presença de cifoses juncionais proximais pré-operatórias, que não evoluíram para a deformidade juncional pós-operatória e ausência completa da anormalidade juncional distal.

\section{REFERÊNCIAS}

1. Helgeson MD, Shah SA, Newton PO, Clements DH 3rd, Betz RR, Marks MC, et al. Evaluation of proximal junctional kyphosis in adolescent idiopathic scoliosis following pedicle screw, hook, or hybrid instrumentation. Spine (Phila Pa 1976). 2010;35(2):177-81.

2. CotrelY, Dubousset J, Guillaumat M. New universal instrumentation in spinal surgery. Clin Orthop Relat Res. 1988;227:10-23.

3. Rothman-Simeone. The Spine. Fifth Edition, Volume I. Chapter 30. 461-465.

4. Kane WJ, Moe JH. A scoliosis-prevalence survey in Minnesota. Clin Orthop Relat Res. 1970;69:216-8

5. Avanzi O, Meves R, Caffaro MFS, Porto FR. Avaliação e comparação da correção no plano sagitale cifose juncional em pacientes com escoliose idiopática do adolescente submetidos a implantes Cotrel-Dubousset e Harri-Luque. Coluna/Columna. 2007;6(4):195-200.

6. Glattes RC, Bridwell KH, Lenke LG, Kim YJ, Rinella A, Edwards C 2nd. Proximal junctional kyphosis in adult spinal deformity following long instrumented posterior spinal fusion: incidence, outcomes, and risk factor analysis. Spine (Phila Pa 1976). 2005;30(14):1643-9.

7. Kim YJ, Lenke LG, Bridwell KH, Kim J, Cho SK, Cheh G, et al. Proximal junctional kyphosis in adolescent idiopathic scoliosis after 3 different types of posterior segmental spinal instrumentation and fusions: incidence and risk factor analysis of 410 cases. Spine (Phila Pa 1976). 2007;32(24):2731-8.

8. Lowe TG, Lenke L, Betz R, Newton P, Clements D, Haher T, et al. Distal junctional kyphosis of adolescent idiopathic thoracic curves following anterior or posterior instrumented fusion: incidence, risk factors, and prevention. Spine (Phila Pa 1976). 2006;31(3):299-302.
9. Cho KJ, Lenke LG, Bridwell KH, Kamiya M, Sides B. Selection of the optimal distal fusion level in posterior instrumentation and fusion for thoracic hyperkyphosis: the sagittal stable vertebra concept. Spine (Phila Pa 1976). 2009;34(8):765-70.

10. Denis $F$, Sun EC, Winter RB. Incidence and risk factors for proximal and distal junctional kyphosis following surgical treatment for Scheuermann kyphosis: minimum five-year follow-up. Spine (Phila Pa 1976). 2009;34(20):E729-34.

11. Kwon BK, Elgafy H, Keynan O, Fisher CG, Boyd MC, Paquette SJ, et al. Progressive junctional kyphosis at the caudal end of lumbar instrumented fusion: etiology, predictors, and treatment. Spine (Phila Pa 1976). 2006:31(17):1943-51.

12. Tveit $P$, Daggfeldt $K$, Hetland $S$, Thorstensson $A$. Erector spinae lever arm length variations with changes in spinal curvature. Spine (Phila Pa 1976). 1994;19(2):199-204.

13. Farcy JP, Schwab FJ. Management of flatback and related kyphotic decompensation syndromes. Spine (Phila Pa 1976). 1997:22(20):2452-7.

14. King HA, Moe JH, Bradford DS, Winter RB. The selection of fusion levels in thoracic idiopathic scoliosis. J Bone Joint Surg Am. 1983;65(9):1302-13.

15. Hollenbeck SM, Glattes RC, Asher MA, Lai SM, Burton DC. The prevalence of increased proximal junctional flexion following posterior instrumentation and arthrodesis for adolescent idiopathic scoliosis. Spine (Phila Pa 1976). 2008;33(15):1675-81.

16. Korovessis P. Sagittal plane analysis of adolescent idiopathic scoliosis. The effect of anterior versus posterior instrumentation. Spine (Phila Pa 1976). 2003;28(14):1624-5; author reply 1625 